\title{
Erratum to: The Effect of Limb Kinematics on the Speed of a Legged Robot on Granular Media
}

\author{
C. Li • P.B. Umbanhowar • H. Komsuoglu • \\ D.I. Goldman
}

Published online: 30 April 2011

(C) Society for Experimental Mechanics 2011

\section{Erratum to: Experimental Mechanics}

\section{DOI 10.1007/s11340-010-9347-1}

In the above mentioned article, due to a systematic error measuring angular position, the penetration force during rotational intrusion, $F_{\mathrm{p}}(\theta)$, was misreported in Fig. $4 \mathrm{~b}$. Correcting the error shifts the angle of peak force $\left(\beta_{0}\right)$ from -0.5 to -0.75 and increases the maximum value of the force increases by $4 \%$ (see figure). Model calculations dependent on $F_{\mathrm{p}}(\theta)$ change slightly with the correction (using $\beta_{0}=-0.75$ and $k^{\prime}=65 \mathrm{~N} / \mathrm{m}$ ) and remain qualitatively unchanged as do the conclusions of the paper.

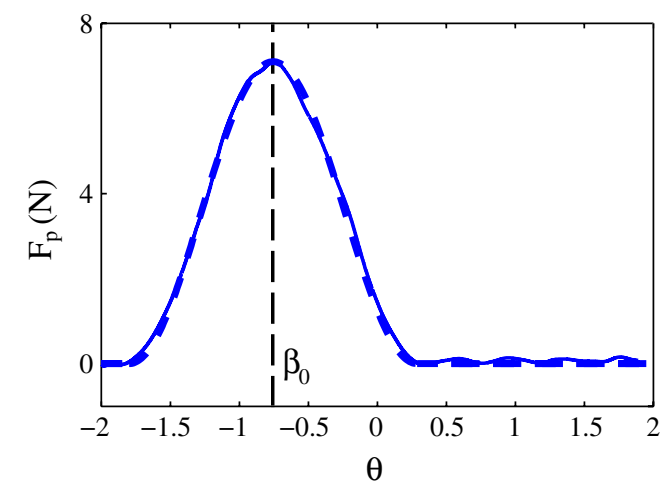

The online version of the original article can be found at http://dx.doi. org/10.1007/s11340-010-9347-1.

C. Li $\cdot$ D.I. Goldman $(\bowtie)$

School of Physics, Georgia Institute of Technology,

Atlanta, GA 30332, USA

e-mail: daniel.goldman@physics.gatech.edu

P.B. Umbanhowar

Department of Mechanical Engineering, Northwestern University,

Evanston, IL 60208, USA

H. Komsuoglu

Department of Electrical and Systems Engineering,

University of Pennsylvania,

Philadelphia, PA 19104, USA 